\section{PP2A plays a key role in inflammation and cancer through tristetraprolin activation}

We have read with great interest the recent work by Ross et al, ${ }^{1}$ which provides novel relevant findings about the therapeutic efficacy of using protein phosphatase 2A (PP2A)-activating drugs to target tristetraprolin (TTP) in rheumatoid arthritis (RA). In this elegant work, the authors showed that TTP is overexpressed and colocalises with activated mitogen-activated protein kinase (MAPK) p38 in RA synovial tissue. MAPK p38 phosphorylates and inhibits TTP at two serine residues, and Ross et al determined that these phosphorylation sites are critical for the role of TTP as a key regulator of inflammatory responses. Since PP2A dephosphorylates TTP at these two serine residues, ${ }^{2}$ they hypothesised and assessed the efficacy of PP2A-activating compounds such as COG1410 and ALL(s) in RA, observing that these agents led to PP2A-mediated TTP activation thereby reducing both inflammation and bone erosion using in vitro and in vivo models of this disease. These results highlight the potential clinical usefulness of PP2A activation as a novel strategy to develop potent anti-inflammatory treatments.

PP2A is a well-known tumour suppressor that has been described commonly inactivated in human cancer. ${ }^{3}$ Moreover, PP2A has been described as a key regulator of the MAPK signalling ${ }^{4}$ and controls the production of proinflammatory chemokines. ${ }^{5}$ Of importance, the risk of developing cancer is higher in people with inflammatory diseases such as colitis or hepatitis. The reason may be that the molecular events generated by the inflammatory response predispose to transformation from chronic inflammation to neoplasia. ${ }^{6}$ Thus, TTP could represent a key linker between inflammation and cancer due to its role as modulator of the expression of both cytokines and proto-oncogenes. ${ }^{7}$ Therefore, the function of PP2A as a TTP activator could be of high relevance and further reinforced by the fact that this phosphatase also targets MAPK signalling, which is responsible for the inhibitory phosphorylation of TTP. $^{5}$

In conclusion, the study by Ross et $a l^{1}$ highlights that PP2A plays a relevant role in inflammation through TTP dephosphorylation and activation. Importantly, these findings would suggest the potential benefits derived from the clinical use of PP2A-activating drugs as anti-inflammatory therapy as well as a novel strategy to prevent cancer development in those patients with chronic inflammatory diseases.
Ion Cristóbal, ${ }^{1}$ Blanca Torrejón, ${ }^{1}$ Juan Madoz-Gúrpide, ${ }^{2}$ Federico Rojo, ${ }^{2}$ Jesús García-Foncillas ${ }^{1}$

${ }^{1}$ Translational Oncology Division, Oncohealth Institute, IIS-Fundacion Jimenez Diaz, UAM, University Hospital 'Fundacion Jimenez Diaz', Madrid, Spain

2Pathology Department, University Hospital 'Fundacion Jimenez Diaz', Autonomous University of Madrid, Madrid, Spain

Correspondence to Dr lon Cristobal and Professor Jesús García-Foncillas, Translational Oncology Division, Oncohealth Institute, IIS-Fundacion Jimenez Diaz, UAM, University Hospital "Fundacion Jimenez Diaz", Avda. Reyes Católicos-2, E-28040 Madrid, Spain; ion.cristobal@fjd.es, jgfoncillas@fjd.es

Contributors IC and BT wrote the manuscript. JM-G, FR and JG-F helped to draft the manuscript.

Funding This work was supported by PI13/02609 and PI15/00934 grants from 'Instituto de Salud Carlos III FEDER'. BT is supported by 'Fundación Conchita Rábago de Jiménez Díaz'.

Competing interests None.

Patient consent Obtained.

Provenance and peer review Not commissioned; internally peer reviewed.

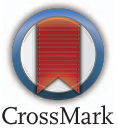

To cite Cristóbal I, Torrejón B, Madoz-Gúrpide J, et al. Ann Rheum Dis 2017;76:e11.

Received 14 October 2016

Accepted 18 October 2016

Published Online First 3 November 2016

Ann Rheum Dis 2017;76:e11. doi:10.1136/annrheumdis-2016-210684

\section{REFERENCES}

1 Ross EA, Naylor AJ, O'Neil JD, et al. Treatment of inflammatory arthritis via targeting of tristetraprolin, a master regulator of pro-inflammatory gene expression. Ann Rheum Dis 2017;76:612-9.

2 Sun L, Stoecklin G, Van Way S, et al. Tristetraprolin (TTP)-14-3-3 complex formation protects TTP from dephosphorylation by protein phosphatase $2 \mathrm{a}$ and stabilizes tumor necrosis factor-alpha mRNA. J Biol Chem 2007:282:3766-77.

3 Sangodkar J, Farrington CC, McClinch K, et al. All roads lead to PP2A: exploiting the therapeutic potential of this phosphatase. FEBS J 2016;283:1004-24.

4 Westermarck J, Hahn WC. Multiple pathways regulated by the tumor suppressor PP2A in transformation. Trends Mol Med 2008;14:152-60.

5 Sandler $\mathrm{H}$, Stoecklin G. Control of mRNA decay by phosphorylation of tristetraprolin. Biochem Soc Trans 2008;36:491-6.

6 Compare D, Nardone O, Nardone G. Non-steroidal anti-inflammatory drugs in the carcinogenesis of the gastrointestinal tract. Pharmaceuticals (Basel) 2010;3:2495-516.

7 Sanduja S, Blanco FF, Young LE, et al. The role of tristetraprolin in cancer and inflammation. Front Biosci (Landmark Ed) 2012;17:174-88. 